\title{
Revision of the North American species of Promicrogaster (Hymenoptera, Braconidae, Microgastrinae), with an updated key to all described species in North and Meso America
}

\author{
Jose Fernandez-Triana' \\ I Canadian National Collection of insects, Ottawa, Canada \\ Corresponding author: Jose Fernandez-Triana (cnc.braconidae@gmail.com)
}

Academic editor: Gavin Broad | Received 18 April 2019 | Accepted 24 May 2019 | Published 28 June 2019

http://zoobank.org/9CAC8BF4-FDD7-4232-9629-E84A4469E955

Citation: Fernandez-Triana J (2019) Revision of the North American species of Promicrogaster (Hymenoptera, Braconidae, Microgastrinae), with an updated key to all described species in North and Meso America. Journal of Hymenoptera Research 70: 89-112. https://doi.org/10.3897/jhr.70.35555

\begin{abstract}
The genus Promicrogaster (Hymenoptera, Braconidae, Microgastrinae) in North America is revised and seven new species are described: floridakeys, gainesvillensis, huachuca, jaymeae, madreanensis, rondeau and virginianus. All but one have so far been found in protected areas of Canada and the USA, with two species collected at rather high altitudes of over 1,600 m.a.s.l. (Arizona, southwestern USA), whereas the other five were found in Eastern North America (southern Ontario and several USA states) at relatively lower altitudes (less than 150 m.a.s.l.). A key to all 31 described species in North and Meso America is provided. A species from Costa Rica previously described in Promicrogaster is here transferred to a different genus as Hypomicrogaster pablouzagai (Fernandez-Triana \& Boudreault, 2016), comb. nov.
\end{abstract}

\section{Keywords}

Microgastrinae, Promicrogaster, Nearctic, taxonomic key

\section{Introduction}

The genus Promicrogaster (Hymenoptera: Braconidae, Microgastrinae) comprises a very distinctive group of microgastrine wasps, on account of its very long, strongly curved ovipositor with its apex sinuate. It is widely distributed in the New World, with 32 currently described species (Fernandez-Triana et al. 2016), and a probable species-richness estimated by Mason (1981) to be over 100 species. In spite of that, specimens are rare in 
collections, and very few details of its biology are presently known. All of the verified host records for Promicrogaster are from caterpillars of the Lepidoptera families Sesiidae and Tineiae living deeply inside more or less woody plant material (reviewed by FernandezTriana et al. 2016), suggesting than the sinuate ovipositor tip is an adaptation to steer it through fissures in harder/more woody structures than mere leaf rolls (e.g., Quicke 2015).

Until now, no species of Promicrogaster had been reported from North America, although Mason (1981) and Fernandez-Triana et al. (2016) mentioned a few undescribed specimens present in collections. Seven sp. nov. from Canada and the United States are described below, increasing the total of known Promicrogaster species in the New World to 38. A recent key to Meso American species by Fernandez-Triana et al. (2016) is updated and modified below to accommodate the new North American species.

\section{Methods}

Promicrogaster is a very rarely collected genus (Mason 1981, Fernandez-Triana et al. 2016), and it is poorly represented in collections. This study is based on 10 specimens available for study in the Canadian National Collection of Insects (CNC) in Ottawa, out of thousands of Nearctic Microgastrinae specimens that were examined.

Morphological terms and measurements of structures are mostly as used by Mason (1981), Huber and Sharkey (1993), Whitfield (1997), Karlsson and Ronquist (2012), and Fernández-Triana et al. (2014a). Mediotergites 1, 2, etc., are abbreviated as T1, T2, etc. Flagellomere 1 is the closest to the pedicel while flagellomere 16 is the apical one. Because the ovipositor in Promicrogaster is curved and crooked at the very apex, its length is difficult to measure accurately; the ovipositor length measurements provided for each sp. nov. are only intended as an approximation. In any case, the ovipositor and its sheaths are some of the longest observed in any Microgastrinae genera; they are usually two times longer than the metatibia length. The descriptions include characters that are commonly used in describing Microgastrinae (e.g., body measurements such as length of body and fore wing, ovipositor sheath; and also color of particular body areas). These characters follow a recent revision of the Mesoamerican Promicrogaster (Fernández-Triana et al. 2016), so as to facilitate future comparisons between species in the New World.

The dichotomous key is based on that provided by Fernández-Triana et al. (2016), expanded to accommodate the new North American species, and revised to correct some previous inaccuracies. In a few couplets of the key, some qualifiers ("either", "and", "or", "and/or") are shown in bold and italic to be explicit that in those cases more than one character state must be considered.

The key mostly uses morphological characters, but in one couplet molecular characters are provided to differentiate species that are morphologically similar to each other. In that case, characteristic loci in the DNA barcoding region are detailed. The bases are numbered from the start of the COI gene according to the reference sequence U37541 (Drosophila melanogaster), and are only diagnostic within that couplet. The letters A, C, $\mathrm{G}$, and $\mathrm{T}$ correspond to adenine, cytosine, guanine, and thymine respectively. 
Photos were taken with a Keyence VHX-1000 Digital Microscope, using a lens with a range of 13-130x. Multiple images through the focal plane were taken of a structure and these were combined to produce a single in-focus image, using the software associated with the Keyence System.

For two species molecular data was available (DNA barcodes, the 5 ' region of the cytochrome c oxidase I (CO1) gene, Hebert et al. (2003)). Information for those sequences can be retrieved from the Barcode of Life Data System (BOLD) (Ratnasingham and Hebert 2007).

\section{Results}

Detailed morphological characterization of Promicrogaster was provided in FernándezTriana et al. (2016). It is a very distinctive genus defined by the combination of elongate and bilobate glossa, large polished areas on lateral face of scutellum, and ovipositor shape and length. Within Microgastrinae, it can only be confused with Sendaphne, but the later has a much less transverse mediotergite 2 , the apex of the ovipositor is not sinuate, the propodeum is entirely or mostly smooth, and the first discal cell is much wider (e.g., Fernández-Triana et al. 2014b).

A total of seven Promicrogaster species, all new and described below, are here recognized for North America (Table 1). All but one have so far been found in protected areas of Canada and the USA. Two species were collected in Southwestern USA (Arizona) at rather high altitudes (over 1,600 m.a.s.l.); the other five were collected in Eastern North America (southern Ontario and several USA states), at relatively lower altitudes (less than 150 m.a.s.l.). Although a few additional species are expected to be found in North America, especially when other collections are studied as comprehensively as that of the $\mathrm{CNC}$ has been, most of the undescribed species in this genus will probably be found in South America.

Five of the seven sp. nov. described below are only represented by a single specimen, in spite of the studied region having tens of thousands of specimens of Microgastrinae in the $\mathrm{CNC}$ collection that were examined for this paper. The relative scarcity of Promicrogaster specimens from North America is similar to what was found when studying the Costa Rican fauna (Fernández-Triana et al. 2016), when very few specimens of the genus were available among tens of thousands of specimens of Microgastrinae examined. Whether Promicrogaster is indeed rare in nature or just not commonly collected is still unknown.

As was the case for species described from Costa Rica (Fernández-Triana et al. 2016), no biological information is available at present for the North American species.

Only two of the sp. nov. (jaymeae and madreanensis) have some molecular data associated (DNA barcodes), in one case only a minibarcode of 165 base pairs.

Further study revealed that one of the species from Costa Rica described in Fernández-Triana et al. (2016), the nominal Promicrogaster pablouzagai Fernandez-Triana \& Boudreault, 2016, is better placed in Hypomicrogaster, with the comb. nov. proposed below (see section "Taxonomic treatment of species, in alphabetical order"), and it is excluded from the key. 
Table I. North American species of Promicrogaster and their known distribution. Data from present paper.

\begin{tabular}{|c|c|}
\hline Species & $\begin{array}{l}\text { Known distribution in North America } \\
\text { (approximate altitude in } \mathbf{m} \text { ) }\end{array}$ \\
\hline Promicrogaster floridakeys Fernandez-Triana, sp. nov. & $\begin{array}{l}\text { USA, Florida, protected areas of the Lower and } \\
\text { Middle Florida Keys, less than } 5 \text { m.a.s.l. }\end{array}$ \\
\hline Promicrogaster gainesvillensis Fernandez-Triana, sp. nov. & USA: Florida, Gainesville, 50 m.a.s.l. \\
\hline Promicrogaster huachuca Fernandez-Triana, sp. nov. & $\begin{array}{l}\text { USA, Arizona, protected area in the Huachuca } \\
\text { Mountains, } 1830 \text { m.a.s.l }\end{array}$ \\
\hline Promicrogaster jaymeae Fernandez-Triana, sp. nov. & $\begin{array}{c}\text { Canada, Ontario, Thousand Islands National } \\
\text { Park, } 120 \text { m.a.s.l; USA: Massachusetts, Blue Hills } \\
\text { Reservation State Park, } 55 \text { m.a.s.l. }\end{array}$ \\
\hline Promicrogaster madreanensis Fernandez-Triana, sp. nov. & $\begin{array}{l}\text { USA, Arizona, protected area in the Patagonia } \\
\text { Mountains, } 1680 \text { m.a.s.l. }\end{array}$ \\
\hline Promicrogaster rondeau Fernandez-Triana, sp. nov. & $\begin{array}{c}\text { Canada, Ontario, Rondeau Provincial Park, } 150 \\
\text { m.a.s.l. }\end{array}$ \\
\hline Promicrogaster virginianus Fernandez-Triana, sp. nov. & USA, Virginia, no further data. \\
\hline
\end{tabular}

The updated key below covers all 32 species so far described from North America and Meso America and believed to be correctly placed in Promicrogaster. Detailed images and full descriptions of the Mesoamerican species are not repeated here as they can be freely accessed in Fernández-Triana et al. (2016). South America is the only remaining area in the New World pending study and description of its species, most of them undescribed.

\section{Key to Promicrogaster species in North and Meso America (female specimens)}

$1 \quad$ Lighter coloured species; clypeus entirely or mostly yellow to orange-yellow; all sternites and hypopygium yellow to orange-yellow; metasoma dorsally with T1-T4 entirely (rarely mostly) yellow, orange or red...........................2

- $\quad$ Darker coloured species; most sternites (usually) and hypopygium (partially to entirely) dark brown; metasoma dorsally mostly dark brown to black (rarely some tergites with small areas orange to light brown); clypeus coloration variable, usually dark brown to black, rarely mostly yellow to orange-yellow .......5

2(1) Propodeum with complete, raised, and strongly defined median carina; and T1 entirely smooth; and malar line more than half eye length; and clypeus large and entirely orange-yellow [Panama, Trinidad]

Promicrogaster miranda Muesebeck, 1958

- $\quad$ Propodeum without a median carina but with central depression; either with T1 mostly rugose and with malar line less than half eye length; or with clypeus smaller and not entirely yellow-red [other Mesoamerican countries, mostly Costa Rica].....

3(2) Metasoma dorsally entirely yellow-orange; T1 with coarse sculpture on 0.7 or more of its length; T2 relatively narrow, its width at posterior margin more 
than $3.0 \times$ its length centrally; scape entirely to mostly black to dark brown [Costa Rica, Honduras, Mexico, Panama, mostly found at elevations over 1,000 m.a.s.l.]. Promicrogaster munda Muesebeck, 1958 Metasoma dorsally with some red, brown or black coloration; T1 comparatively smoother, at most with sculpture on posterior 0.5 of its length; T2 broader, its width at posterior margin usually less than $3.0 \times$ its length centrally; scape mostly yellow [Costa Rica (ACG), dry forest, under 300 m.a.s.l.]................. 4

4(3) Posterior margin of clypeus strongly concave; lower face clearly elongate, malar line at least $1.5 \times$ mandible width; fore wing vein $1 \mathrm{M}$ transparent; $\mathrm{T} 1$ shape more or less rectangular (width at middle length, anterior and posterior margins about the same); T1-T4 entirely orange-yellow, T5+ mostly dark brown to black

Promicrogaster fabriciocambroneroi Fernandez-Triana \& Boudreault, 2016

- $\quad$ Posterior margin of clypeus very slightly concave, almost straight; lower face of normal length, malar line $1.0 \times$ mandible width; fore wing with vein $1 \mathrm{M}$ brown; T1 narrowing towards posterior margin; T1 mostly red with black margins (posterior 0.3 of 11 sometimes black), T2-T4 red-orange or yelloworange, $\mathrm{T} 5+$ yellow with small central band brown

..... Promicrogaster alexmartinezi Fernandez-Triana \& Boudreault, 2016

5(1) Antenna with most of flagellomeres 10-16 yellow-white .6

- $\quad$ Antenna entirely dark brown to black (rarely with flagellomeres 1-4 lighter in colour than rest of antenna).

6(5) Antenna with flagellomeres 11-16 yellow-white; propleuron, anterior 0.5 of metacoxa, sternites and hypopygium dark brown; ovipositor strongly down curved on posterior 0.2 [Costa Rica (ACG), cloud forest over $1000 \mathrm{~m}$ ]

...... Promicrogaster leilycastilloae Fernandez-Triana \& Boudreault, 2016 Antenna with flagellomeres $1-8$ and 15-16 dark brown, and flagellomeres 9-14 (sometimes only 10-14) yellow white; propleuron, metacoxa, sternites and hypopygium mostly yellow to orange-yellow; ovipositor relatively straight [Costa Rica (ACG), mid-elevation rain forests, 500-800m]

..Promicrogaster daretrizoi Fernandez-Triana \& Boudreault, 2016

7(5) Fore wing without areolet, and hypopygium and sternites almost always entirely dark brown (at most hypopygium with small pale spot basally, and/or anterior 2-3 sternites yellow), and smaller size (body length $1.8-2.4 \mathrm{~mm}$, fore wing length $2.0-2.6 \mathrm{~mm}$ )

- $\quad$ Fore wing with small areolet, and/or hypopygium and sternites usually mostly to entirely yellow, and/or larger size (body and fore wing lengths usually $3.0-4.0 \mathrm{~mm}$ )

8(7) Propodeum mostly sculptured (except for polished areas postero-laterally) .....9

- $\quad$ Propodeum mostly smooth (except for small striae around nucha)............14

9(8) Propodeum with almost complete areola (clearly defined posteriorly by carinae, definition anteriorly more obscured by surrounding sculpture); T2 mostly smooth [United States (Florida Keys), mangrove-hammock forest 
transition, at around sea level]

Promicrogaster floridakeys Fernandez-Triana, sp. nov.

- $\quad$ Propodeum without defined areola (with longitudinal sculpture, mostly near posterior half of propodeum); T2 mostly sculptured, except for smooth central area [species either from Canada or from Costa Rica, found at much higher altitudes and/or completely different ecosystems]....

10(9) Ocelli comparatively smaller: ocular-ocellar line $2.8 \times$ posterior ocellus diameter, ocular-ocellar line $2.0 \times$ interocellar distance; ovipositor length 2.0 $\times$ metatibia length [Canada (Ontario), $42^{\circ} \mathrm{N}$, sand dunes, at around 170 m.a.s.l.] Promicrogaster rondeau Fernandez-Triana, sp. nov. Ocelli comparatively larger: ocular-ocellar line 1.6-2.0 $\times$ (very rarely $2.3 \times$ ) posterior ocellus diameter, ocular-ocellar line 0.9-1.6 $\times$ interocellar distance; ovipositor length more than $2.0 \times$ metatibia length [Costa Rica (ACG), 10$11^{\circ} \mathrm{N}$, rainforest, at $1,000-1,100$ m.a.s.l.)]

11(10) Smaller size, body length $2.0-2.1 \mathrm{~mm}$, fore wing length $2.2 \mathrm{~mm}$, metacoxa 0.45-0.46 mm; ocular-ocellar line 0.08-0.09 mm; T1 width/length 0.4 $0.5 \times$; ten diagnostic characters in the DNA barcoding region: $79 \mathrm{C}, 235 \mathrm{C}$, 346C, 364C, 386A, 415A, 421G, 562A, 607G, 622C

Promicrogaster fabiancastroi Fernandez-Triana \& Boudreault, 2016 Larger size, body length $2.3-2.4 \mathrm{~mm}$, fore wing length $2.4-2.6 \mathrm{~mm}$, metacoxa $0.47-0.56 \mathrm{~mm}$; ocular-ocellar line $0.11-0.15 \mathrm{~mm}$ (rarely 0.08 ); T1 width/length $0.5-0.7 \times$; different base pairs in the barcoding region: $79 \mathrm{~A}$ or $79 \mathrm{~T}, 235 \mathrm{~T}, 346 \mathrm{~A}$ or $346 \mathrm{~T}, 364 \mathrm{~A}$ or $364 \mathrm{~T}, 386 \mathrm{~T}, 415 \mathrm{G}, 421 \mathrm{~A}$ or $421 \mathrm{~T}, 562 \mathrm{G}$ or $562 \mathrm{~T}, 607 \mathrm{~A}$ or $607 \mathrm{~T}, 622 \mathrm{~A}$ or $622 \mathrm{~T}$

12(11) Ocular-ocellar line shorter than interocellar distance $(0.9 \times)$; T1 posterior width $1.3 \times$ T2 central length; T2 width $3.2 \times$ its length centrally

Promicrogaster luismendezi Fernandez-Triana \& Boudreault, 2016 Ocular-ocellar line longer than interocellar distance $(1.4-1.6 \times)$; T1 posterior width 1.8-2.0 × T2 central length; T2 width 3.5-4.9 $\times$ (usually more than $4.0 \times$ ) its length centrally

13(12) T2 width $4.9 \times$ its length centrally; T1 length $1.9 \times$ its posterior width; larger species, fore wing length $2.6 \mathrm{~mm}$, metacoxa length $0.6 \mathrm{~mm}$, metafemur length $0.7 \mathrm{~mm}$, metatibia $0.9 \mathrm{~mm}$

Promicrogaster eddycastroi Fernandez-Triana \& Boudreault, 2016 T2 width 3.5-4.0 $\times$ its length centrally; T1 length $1.5-1.7 \times$ its posterior width; smaller species, fore wing length $2.3-2.4 \mathrm{~mm}$, metacoxa length 0.5 $\mathrm{mm}$, metafemur length $0.5-0.6 \mathrm{~mm}$, metatibia $0.7-0.8 \mathrm{~mm}$

... Promicrogaster naomiduarteae Fernandez-Triana \& Boudreault, 2016

14(8) Pterostigma with anterior 0.3 or more white, most veins of fore wing transparent or white [Costa Rica (ACG), dry forest under 300 m.a.s.l.]

Promicrogaster daniellopezi Fernandez-Triana \& Boudreault, 2016 Pterostigma entirely brown or at most with anterior 0.1 pale, fore wing with veins mostly brown [Costa Rica (ACG), cloud forests over 1,000 m.a.s.l.] ... 
15(14) Posterior 0.6 of T1 sculptured; clypeus, procoxa, most of sternites and laterotergites brown

....... Promicrogaster ronycastilloi Fernandez-Triana \& Boudreault, 2016

- $\quad$ Posterior 0.6 of T1 mostly smooth (at most with sculpture restricted to margins); labrum yellow-orange, procoxa yellow, sternites and laterotergites mostly to partially yellow

\section{...Promicrogaster sebastiancambroneroi Fernandez-Triana \& Boudreault, 2016}

16(7) Posterolateral corners of anteromesoscutum orange; hypopygium and sternites dark brown; T1 hardly narrowing towards posterior margins; T1 relatively wide, its medial length barely longer than its width at anterior margin [Panama] Promicrogaster polyporicola Muesebeck, 1958

- $\quad$ Anteromesoscutum entirely black; hypopygium and sternites usually mostly to entirely yellow; T1 usually narrowing towards posterior margin; T1 relatively narrower, its medial length much longer than its width at anterior margin [Brazil, Canada, Costa Rica, Mexico, United States]

17(16) Propleuron almost entirely yellow, except for anterior 0.2 near head yellowwhite (rarely propleuron partially yellow, partially light-brown); hypopygium, sternites and most laterotergites entirely yellow (hypopygium may have a dark spot on posterior 0.1 or less).

Propleuron almost entirely dark brown to black, except for anterior 0.2 near head yellow; hypopygium, sternites and laterotergites at least partially dark brown

18(17) Metacoxa dark brown on anterior 0.6; tegula brown; propleuron partially yellow, partially light-brown

... Promicrogaster kiralycastilloae Fernandez-Triana \& Boudreault, 2016 Metacoxa, tegula and propleuron entirely yellow (propleuron with anterior 0.2 near head yellow-white)

19(18) T3 mostly yellow-white, with anterior 0.4 light brown, T4-7 brown anteriorly, white on posterior 0.3-0.5 [Costa Rica (ACG), cloud forest over 1,000 m.a.s.1.].

... Promicrogaster kevinmartinezi Fernandez-Triana \& Boudreault, 2016 All tergites dark brown to black [Costa Rica (ACG), mid-elevation rain forest, 500 m.a.s.l.].

.... Promicrogaster eimyobandoae Fernandez-Triana \& Boudreault, 2016

20(17) Fore wing without areolet

... Promicrogaster andreyvallejosi Fernandez-Triana \& Boudreault, 2016

Fore wing with small areolet

21(20) Flagellomeres 1-4 yellow-brown, clearly paler than rest of entirely brown flagellomeres ; orange-yellow areas on metapleuron posterior 0.4 , T1 anterior 0.6 , T3 anterior 0.5 and small spot on mesopleuron posteriorly.

Promicrogaster hillaryvillafuerteae Fernandez-Triana \& Boudreault, 2016 All flagellomeres same color (brown to dark brown); meso- and metapleuron entirely dark brown to black, coloration of T1 and T3 variable but not as above 
22(21) Tegula and wing base dark brown to black; clypeus black (same color as face); clypeus slightly protruding and labrum slightly depressed, giving the appearance of a circular opening between the margin of clypeus and mandibles...... ... Promicrogaster monteverdensis Fernandez-Triana \& Boudreault, 2016

- $\quad$ Tegula and wing base yellow; clypeus entirely to partially orange-yellow or orange-brown (clearer than face); clypeus not protruding and labrum not depressed, not giving the appearance of a circular opening.

23(22) Flagellomere 15 length 1.6-1.7 $\times$ its width; and ovipositor tip strongly bent downwards; and clypeus entirely orange-yellow (different from dark brown to black face); and body length and fore wing length $4.0 \mathrm{~mm}$; and $\mathrm{T} 1 \mathrm{al}-$ most parallel-sided, very slightly narrowing towards posterior margin; and T2 mostly sculptured; and T3 with small yellow spot laterally [Brazil, Mexico]...

Promicrogaster apharea Nixon, 1965

- $\quad$ Flagellomere 15 length 1.0-1.3 $\times$ its width; and/or ovipositor tip less strongly bent downwards; and/or clypeus entirely to partially dark brown to black (same color as face); and/or body length and fore wing length less than $4.0 \mathrm{~mm}$; and/or T1 clearly narrowing towards posterior margin; and/or T2 mostly smooth; and/ or T3 entirely dark brown to black [Canada, Costa Rica, United States] ..........24

24(23) Metacoxa with at least anterior 0.6 (usually more) dark brown to black [United States; Costa Rican specimens running through this couplet come from cloud forests over 1,000 m.a.s.l. in ACG]

- $\quad$ Metacoxa mostly to entirely yellow (at most with small, dark spot on anterior 0.1-0.3 dorsally) [United States; Costa Rican specimens running through this couplet come from dry forest or mid-elevation rainforests at 300-500

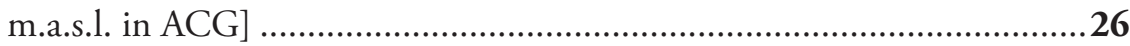

25(24) Smaller ocelli, ocular-ocellar line $2.6 \times$ as long as posterior ocellus diameter; more rounded head, its maximum width $1.15 \times$ its height; smaller body length $(2.5 \mathrm{~mm})$ and fore wing length $(2.6 \mathrm{~mm})$; tegula yellow [United States] ..................Promicrogaster virginianus Fernandez-Triana, sp. nov. Bigger ocelli, ocular-ocellar line 1.9-2.0 $\times$ as long as posterior ocellus diameter; more ovoid head, its maximum width $1.3 \times$ its height; larger body length $(3.3-3.5 \mathrm{~mm})$ and fore wing length $(3.7 \mathrm{~mm})$; tegula brown [Costa Rica (ACG), cloud forests over 1,000 m.a.s.l.]

Promicrogaster brandondinartei Fernandez-Triana \& Boudreault, 2016*

26(24) T3 with yellow spots laterally; T2 mostly sculptured (but sometimes smooth centrally) and/or $\mathrm{T} 1$ clearly narrowing towards posterior margin, its length at least $2.0 \times$ its width at posterior margin 27 T3 entirely dark brown to black; T2 smooth and T1 usually barrel-shaped to almost parallel-sided, rarely slightly narrowing towards posterior margin, its length $1.7 \times$ its width at posterior margin .....

* In the original description of this species the color of metacoxa was wrongly described as 'yellow' but after re-examining all specimens is evident that was an error, as all specimens have metacoxa with anterior 0.5-0.6 dark brown to black 
27(26) Fore wing vein R1 pale (yellow-white); metasoma with T3+ yellow-orange on posterior 0.2-0.5 [Canada (ON); United States (Massachusetts); $42-44^{\circ} \mathrm{N}$ ].

Promicrogaster jaymeae Fernandez-Triana, sp. nov.

- $\quad$ Fore wing vein R1 dark brown; metasoma mostly dark brown to black, at most T3 with yellow spots laterally [Costa Rica (ACG); United States (AZ), $\left.31^{\circ} \mathrm{N}\right]$

28(27) Clypeus almost entirely black (at most with very small yellow spots on postero-lateral margins); fore wing with most veins dark brown; hypopygium partially yellow; ovipositor length 2.50-2.60 $\times$ metatibia length; tip of antenna with shorter flagellomeres (flagellomere 14 1.3-1.4 $\times$ as long as wide, flagellomere $151.2 \times$ as long as wide) [Costa Rica (ACG), 280-300 m.a.s.l.]

Promicrogaster liagrantae Fernandez-Triana \& Boudreault, 2016

- $\quad$ Clypeus entirely to mostly orange; fore wing with most veins light yellow to almost transparent; hypopygium dark brown to brown; ovipositor length 1.97-2.14 $\times$ metatibia length; tip of antenna (when known) with longer flagellomeres (flagellomere $141.6 \times$ as long as wide, flagellomere $151.5 \times$ as long as wide) [United States]

29

29(28) Comparatively smaller species (body length: $3.10 \mathrm{~mm}$, fore wing length: $3.00 \mathrm{~mm}$ ); T1 length $1.9 \times$ its width at posterior margin; T2 posterior margin width $3.2 \times$ it length medially and slightly more sculptured; head and mesosoma mostly dark reddish-brown; fore wing areolet almost obliterated [Florida, 50 m.a.s.l.]

Promicrogaster gainesvillensis Fernandez-Triana, sp. nov.

- $\quad$ Comparatively larger species (body length: $3.70 \mathrm{~mm}$, fore wing length: 3.80 $\mathrm{mm}$ ); T1 length $2.7 \times$ its width at posterior margin; T2 posterior margin width $4.4 \times$ its length medially and mostly smooth (except for sculpture near posterior margin); head and mesosoma mostly black; fore wing areolet clearly visible and defined [Arizona (Huachuca Mountains), 1830 m.a.s.l.]

Promicrogaster huachuca Fernandez-Triana, sp. nov.

30(26) Clypeus with strong notch centrally on posterior margin; T1 and T2 almost entirely smooth; metapleuron relatively more sculptured on posterior 0.4; body and fore wing length over $4.5 \mathrm{~mm}$ [United States (AZ, Patagonia Mountains), 1,675 m.a.s.1.]

Promicrogaster madreanensis Fernandez-Triana, sp. nov. Clypeus without notch centrally on posterior margin; T1 with some sculpture on posterior $0.4-0.5$, T2 with some punctuation on margins; metapleuron relatively less sculptured, almost smooth on posterior 0.4 ; body and fore wing lengths 3.6-4.0 mm [Costa Rica (ACG), 400-520 m.a.s.l.]

.... Promicrogaster tracyvindasae Fernandez-Triana \& Boudreault, 2016 


\section{Taxonomic treatment of species, in alphabetical order}

Hypomicrogaster pablouzagai (Fernandez-Triana \& Boudreault, 2016), comb. nov.

Promicrogaster pablouzagai Fernandez-Triana \& Boudreault, 2016.

Notes. A critical re-examination of the available specimens (including the holotype) as well as the numerous DNA barcodes available, clearly indicate that this species is better placed within Hypomicrogaster. The propodeum has an irregular pattern of carinae around the median longitudinal carina, but still it is possible to distinguish a partially defined areola (at least apically) which is similar to other known species of Hypomicrogaster. The head is also considerably transverse (i.e., significantly much wider than high in frontal view), another morphological feature that is typical in this genus. The DNA barcodes cluster close to many other species of Hypomicrogaster and relatively distant from Promicrogaster, further supporting transferring the species here.

\section{Promicrogaster floridakeys Fernandez-Triana, sp. nov.} http://zoobank.org/24EBD28C-19D2-4FAE-8DA5-10E26CB39103

Fig. 1 A-G

Material examined. Holotype. UNITED STATES • + , CNC; Florida, Long Key State Park; 24.8127N, 80.8231W; 24 Aug-26 Oct 2016; J McIntosh leg; Malaise trap; CNC634252.

Paratypes. UNITED STATES • + , CNC; Florida, Monroe County, Middle Torch Key; 1-30 Jun 1985; S \& J Peck leg; Malaise trap; mangrove-hardwood transition; CNC505706 • +, CNC; Florida, Monroe County, Big Pine Key; 1-30 Mar 1986; S \& J Peck leg; Malaise trap; CNC526849.

Diagnosis. Among the darker coloured species with unicolorous (brown to black) flagellomeres, P. floridakeys is unique in having the propodeum with an almost complete areola (all other species lack an areola).

Description. Head: mostly black, labrum and mandibles yellow. Flagellomeres: dark brown. Mesosoma: black. Tegula: yellow. Metasoma (dorsally): black to dark brown. Metacoxa: mostly black to dark brown (posterior $0.1-0.2$ yellow). Malar distance: less than $0.2 \times$ eye length. Fore wing areolet: absent. T1 sculpture: anterior 0.5 smooth, posterior 0.5 sculptured. T2 sculpture: mostly smooth. Body length: $2.30-2.50$ $\mathrm{mm}$. Fore wing length: $2.40-2.55 \mathrm{~mm}$. Ovipositor length: $1.50-1.70 \mathrm{~mm}$. Ocularocellar line: $0.12 \mathrm{~mm}$. Interocellar distance: $0.09 \mathrm{~mm}$. Posterior ocellus diameter: 0.06 $\mathrm{mm}$. Metacoxa length: $0.51-0.56 \mathrm{~mm}$. Metafemur length: $0.61-0.65 \mathrm{~mm}$. Metatibia length: $0.71-0.77 \mathrm{~mm}$. T1 length/width at posterior margin: $0.33-0.35 \mathrm{~mm} / 0.14$ $\mathrm{mm}$. T2 length/width at posterior margin: $0.07-0.08 \mathrm{~mm} / 0.30-0.32 \mathrm{~mm}$.

Distribution. UNITED STATES, Florida, protected areas of the Lower and Middle Florida Keys (less than 5 m.a.s.l.). Specimens have been caught at mangrove or hardwood habitats. 

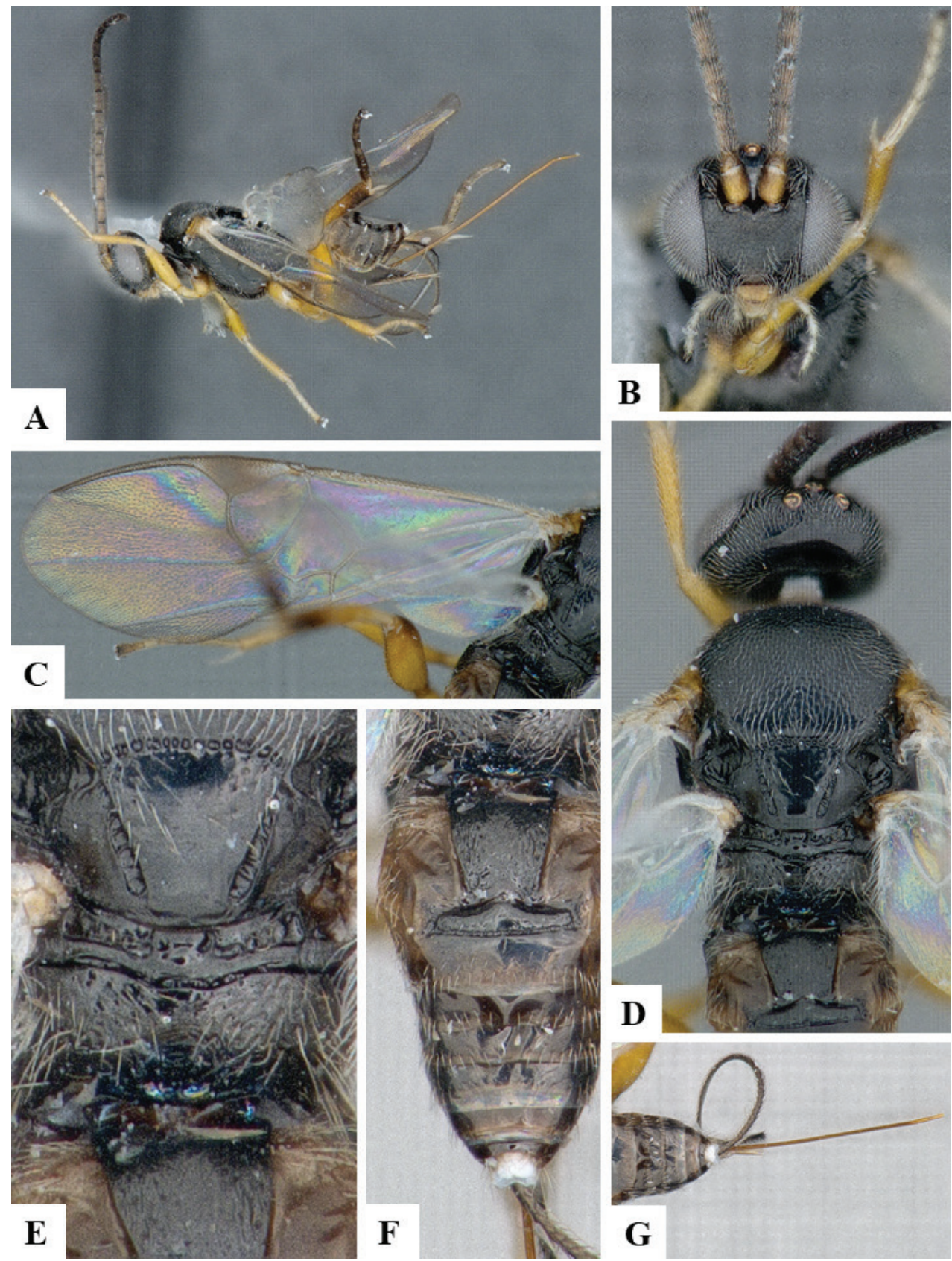

Figure I. Promicrogaster floridakeys, female holotype. A Habitus, lateral $\mathbf{B}$ head, frontal $\mathbf{C}$ fore wing $\mathbf{D}$ head and mesosoma, dorsal $\mathbf{E}$ scutellar complex and propodeum, dorsal $\mathbf{F}$ metasoma, dorsal $\mathbf{G}$ ovipositor.

Biology. Unknown.

Molecular data. Not available.

Etymology. Named after the Florida Keys, with the intention to recognize the beautiful yet fragile ecosystems present there. 


\section{Promicrogaster gainesvillensis Fernandez-Triana, sp. nov. \\ http://zoobank.org/9AD19A17-D8CB-411D-A150-D820C342AA18}

Fig. 2 A-E

Material examined. Holotype. UNITED STATES • + , CNC; Florida, Gainesville, Doyle Conner Building; 09 Oct 1973; E.E. Grissell leg; Malaise trap; CNCHYM 01987.

Diagnosis. This species is morphologically similar to P. huachuca (Arizona), P. jaymeae (Canada), and $P$. liagrantae (Costa Rica), but there are enough morphological differences (see key above for details on how to separate those four species) and disparate geographical distributions (including different ecosystems and considerable variation in altitude) to consider all of them as different.

Description. Head: mostly dark reddish-brown, labrum and mandibles yellow, clypeus mostly dark reddish-brown but with comparatively large yellow spots on postero-lateral margins. Flagellomeres: dark brown. Mesosoma: mostly dark reddish-brown laterally, dorsally mostly black (but with faint reddish-brown spots on posterolateral margins of anteromesoscutum). Tegula: yellow. Metasoma (dorsally): mostly dark brown to black, T3 with yellow spots laterally. Metacoxa: yellow. Malar distance: less than $0.2 \times$ eye length. Fore wing areolet: present. T1 sculpture: anterior 0.5 smooth, posterior 0.5 sculptured. T2 sculpture: mostly sculptured, except for smooth central area. Body length: $3.10 \mathrm{~mm}$. Fore wing length: $3.00 \mathrm{~mm}$. Ovipositor length: $2.25 \mathrm{~mm}$. Ocular-ocellar line: $0.15 \mathrm{~mm}$. Interocellar distance: $0.08 \mathrm{~mm}$. Posterior ocellus diameter: $0.07 \mathrm{~mm}$. Metacoxa length: 0.75 $\mathrm{mm}$. Metafemur length: $0.85 \mathrm{~mm}$. Metatibia length: $1.05 \mathrm{~mm}$. T1 length/width at posterior margin: $0.48 \mathrm{~mm} / 0.25 \mathrm{~mm}$. T2 length/width at posterior margin: 0.12 $\mathrm{mm} / 0.38 \mathrm{~mm}$.

Distribution. UNITED STATES, Florida, Gainesville, 50 m.a.s.l.

Biology. Unknown.

Molecular data. Not available.

Etymology. Named after the type locality, as a recognition to the important insect collections hosted in that city and the vibrant entomological community living there.

\section{Promicrogaster huachuca Fernandez-Triana, sp. nov.}

http://zoobank.org/4DE29D6C-4FBA-47F9-A345-A62300595942

Fig. $3 \mathrm{~A}-\mathrm{F}$

Material examined. Holotype. UNITED STATES $\bullet+$, CNC; Arizona, Sierra Vista, Huachua Mountains, Ramsey Canyon; 1829 m.a.sl.; 07 Nov 1967; Sternitzky leg; CNCHYM 01988.

Diagnosis. This species is morphologically similar to P. gainesvillensis (Florida), P. jaymeae (Canada), and P. liagrantae (Costa Rica), but there are enough morphological differences (see key above for details on how to separate those four species) and disparate geographical distributions (including different ecosystems and considerable variation in altitude) to consider all of them as different. 

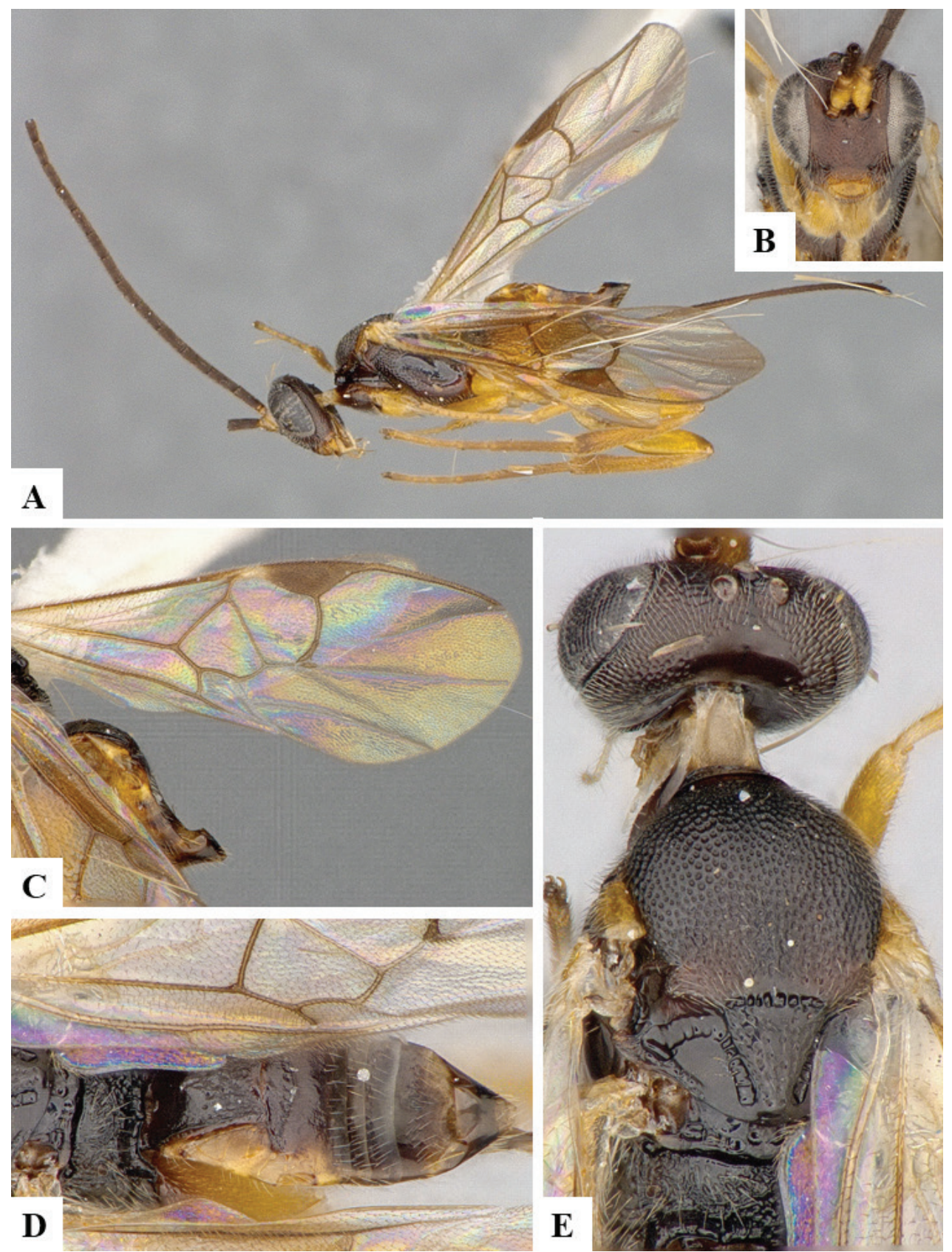

Figure 2. Promicrogaster gainesvillensis, female holotype. A Habitus, lateral B head, frontal $\mathbf{C}$ fore wing D propodeum and metasoma, dorsal $\mathbf{E}$ head and mesosoma, dorsal.

Description. Head: mostly black, labrum and mandibles yellow. Flagellomeres: dark brown. Mesosoma: black. Tegula: yellow. Metasoma (dorsally): mostly dark brown to black, T3 with yellow spots laterally. Metacoxa: yellow. Malar distance: $0.3 \times$ eye length. 

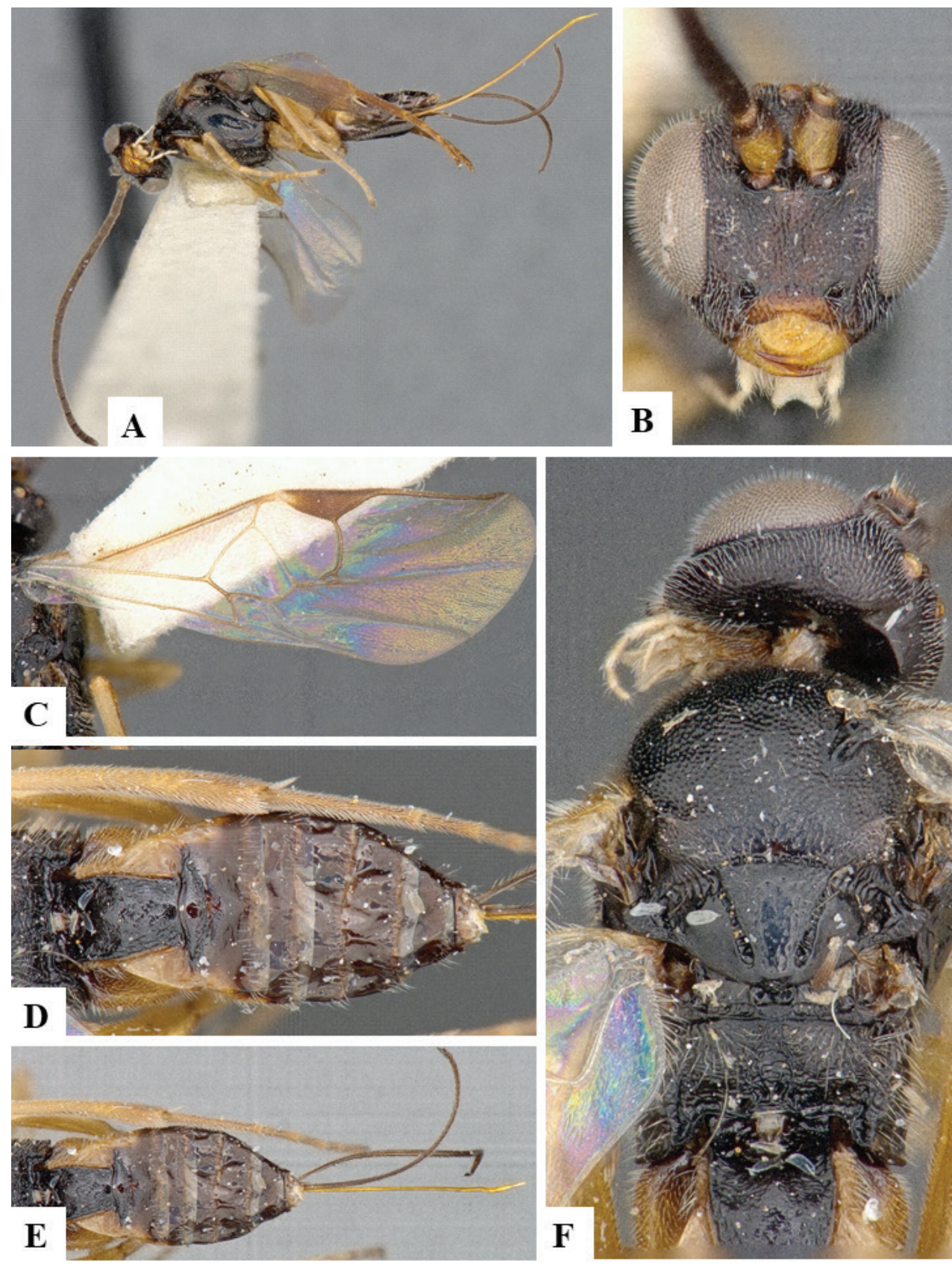

$\mathbf{F}$

Figure 3. Promicrogaster huachuca, female holotype. A Habitus, lateral B head, frontal C fore wing $\mathbf{D}$ propodeum and metasoma, dorsal $\mathbf{E}$ ovipositor $\mathbf{F}$ head and mesosoma, dorsal.

Fore wing areolet: present. T1 sculpture: anterior 0.5 smooth, posterior 0.5 sculptured. T2 sculpture: mostly smooth but with sculpture near margins. Body length: $3.70 \mathrm{~mm}$. Fore wing length: $3.80 \mathrm{~mm}$. Ovipositor length: $2.40 \mathrm{~mm}$. Ocular-ocellar line: $0.17 \mathrm{~mm}$. 
Interocellar distance: $0.10 \mathrm{~mm}$. Posterior ocellus diameter: $0.09 \mathrm{~mm}$. Metacoxa length: $0.85 \mathrm{~mm}$. Metafemur length: $0.95 \mathrm{~mm}$. Metatibia length: 1.22 . T1 length/width at posterior margin: $0.60 / 0.22 \mathrm{~mm}$. T2 length/width at posterior margin: $0.12 \mathrm{~mm} / 0.53 \mathrm{~mm}$.

Distribution. UNITED STATES, Arizona, Huachuca Mountains, 1,829 m.a.s.l.

Biology. Unknown.

Molecular data. Not available.

Etymology. Named after the Huachuca Mountains, one of the major Madrean sky island ranges in Arizona. The Madrean sky islands are pine-oak woodlands found at higher elevations in Mexico, Arizona and New Mexico (USA); they are surrounded at lower elevations by the Sonoran and Chihuahuan deserts, and are important because of their endemism, and relict populations.

\section{Promicrogaster jaymeae Fernandez-Triana, sp. nov.} http://zoobank.org/D5ED2A45-7F43-4B1F-9813-88B7E73BB9E0 Figs 4 A-F, 5 A-G

Material examined. Holotype. CANADA • + , CNC; Ontario; St. Lawrence Islands National Park; Jones Creek by Mallory Town; Country Road 5; 44.4747N, 75.8652W; 117 m.a.s.l; 19 Sep 2012; Mixed forest - sugar maple and white birch; St. Lawrence Islands National Park leg; BIOUG03887-G02.

Paratype. UNITED STATES • $q$, MCZ (Museum of Comparative Zoology, Harvard University, Cambridge, USA); Massachusetts, Blue Hills Reservation, Milton; 19 Aug 1963; H.E. Evans leg; CNC666703.

Diagnosis. This species is morphologically similar to $P$. gainesvillensis (Florida), $P$. huachuca (Arizona), and P. liagrantae (Costa Rica), but there are enough morphological differences (see key above for details on how to separate those four species) and disparate geographical distributions (including different ecosystems and considerable variation in altitude) to consider all of them as different. The Canadian species can be separated based on its fore wing vein R1 yellow-white, T3+ yellow-orange on posterior $0.2-0.5$, metacoxa dark brown on anterior 0.3 , and T2 mostly sculptured (fore wing vein R1 dark brown; metasoma mostly dark brown to black, metacoxa entirely yellow, and T2 mostly smooth on P. liagrantae).

Description. Head: mostly black, labrum and mandibles yellow. Flagellomeres: dark brown. Mesosoma: black. Tegula: yellow. Metasoma (dorsally): mostly dark brown, with T3+ yellow-orange on posterior 0.2-0.5. Metacoxa: mostly orange yellow, with black to dark brown spot on anterior 0.3 dorsally. Malar distance: less than $0.2 \times$ eye length. Fore wing areolet: present. T1 sculpture: mostly sculptured. T2 sculpture: mostly smooth. Body length: 3.40-3.50 mm. Fore wing length: $3.40 \mathrm{~mm}$. Ovipositor length: 2.10-2.30 mm. Ocular-ocellar line: $0.14 \mathrm{~mm}$. Interocellar distance: 0.09-0.10 $\mathrm{mm}$. Posterior ocellus diameter: 0.08-0.09 mm. Metacoxa length: 0.80-0.90 mm. Metafemur length: 0.90-1.02 mm. Metatibia length: 1.15-1.28. T1 length/width at posterior margin: $0.47-0.57 / 0.29 \mathrm{~mm}$. T2 length/width at posterior margin: 0.11 $\mathrm{mm} / 0.42-0.43 \mathrm{~mm}$. 

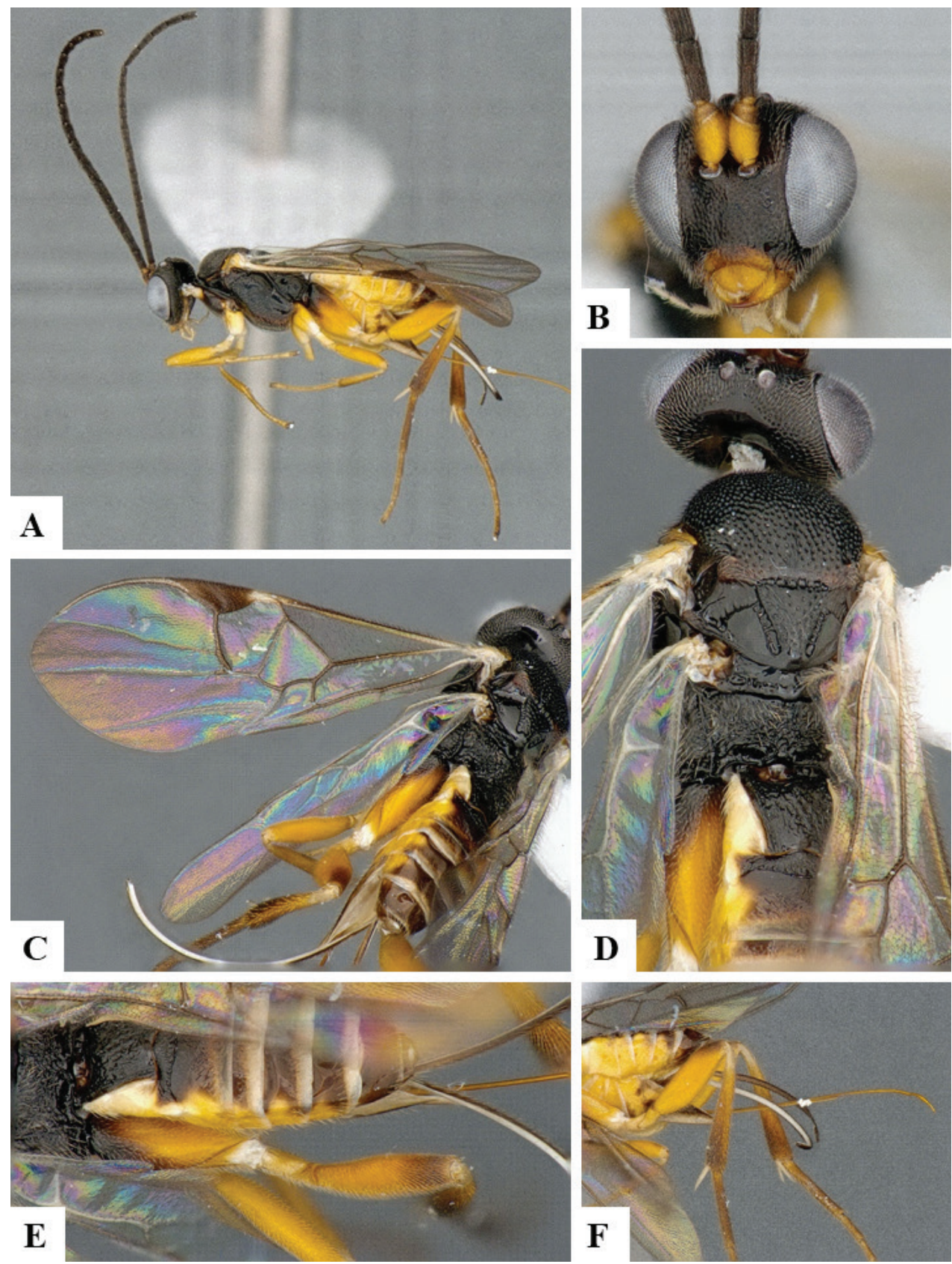

Figure 4. Promicrogaster jaymeae, female holotype. A Habitus, lateral $\mathbf{B}$ head, frontal $\mathbf{C}$ fore wing $\mathbf{D}$ head and mesosoma, dorsal $\mathbf{E}$ propodeum and metasoma, dorsal $\mathbf{F}$ ovipositor.

Distribution. CANADA, Ontario; UNITED STATES, Florida, Massachusetts. Collected between 55 and 117 m.a.s.l.

Biology. Unknown. 


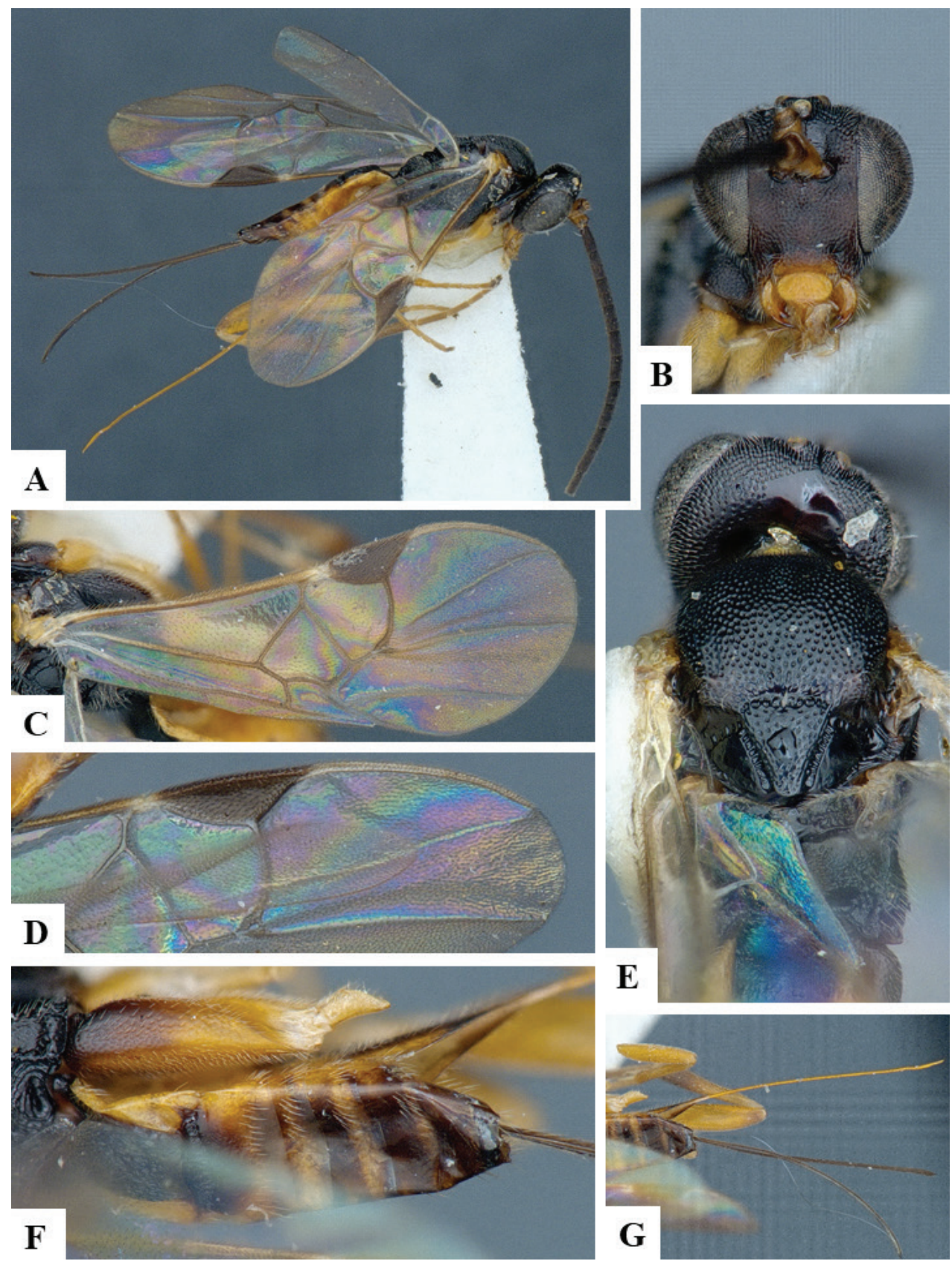

Figure 5. Promicrogaster jaymeae, female paratype. A Habitus, lateral $\mathbf{B}$ head, frontal $\mathbf{C}$ fore wing $\mathbf{D}$ details of pterostigma and fore wing areolet $\mathbf{E}$ head and mesosoma, dorsal $\mathbf{F}$ metasoma, dorsal $\mathbf{G}$ ovipositor.

Molecular data. The holotype rendered an almost complete DNA barcode (622 base pairs), the sequence page in BOLD is CNSLK014-12. It belongs to BIN BOLD:ADA9820, which contains another sequence, from P. liagrantae (Costa Rica). 
The two sequences are rather similar ( $1.73 \%$ base pairs difference) but represent different species.

Etymology. Named after Jayme Sones, Collections Manager of the Centre for Biodiversity Genomics (CBG), University of Guelph, Canada, in appreciation of the extraordinary work she has done for the $\mathrm{CBG}$ during the past 15 years, and as a big thanks for her support and help with many braconid loans and other topics over the years.

\section{Promicrogaster madreanensis Fernandez-Triana, sp. nov. http://zoobank.org/C3EE1313-A3D8-4865-96EA-3C5B9A620D38} Fig. $6 \mathrm{~A}-\mathrm{F}$

Material examined. Holotype. UNITED STATES • + , CNC; Arizona, Patagonia Mountains; 1676 m.a.sl.; 10 Jul 1962; H.E. Milliron leg; CNCHYM 01986.

Diagnosis. This species is morphologically similar to P. tracyvindasae (Costa Rica), but can be distinguished from it (and from all other described species of Promicrogaster from North and Meso America) because of its clypeus with a strong notch centrally on posterior margin.

Description. Head: mostly dark reddish-brown, labrum and mandibles yellow, clypeus orange-yellow. Flagellomeres: dark brown. Mesosoma: mostly black but ventrolaterally reddish-brown. Tegula: yellow. Metasoma (dorsally): mostly dark brown to black. Metacoxa: yellow. Malar distance: $0.3 \times$ eye length. Fore wing areolet: present. T1 sculpture: mostly smooth. T2 sculpture: mostly smooth but with sculpture near margins. Body length: $4.90 \mathrm{~mm}$. Fore wing length: $4.90 \mathrm{~mm}$. Ovipositor length: approximately $2.90 \mathrm{~mm}$. Ocular-ocellar line: $0.20 \mathrm{~mm}$. Interocellar distance: $0.12 \mathrm{~mm}$. Posterior ocellus diameter: $0.10 \mathrm{~mm}$. Metacoxa length: $1.10 \mathrm{~mm}$. Metafemur length: $1.20 \mathrm{~mm}$. Metatibia length: 1.50 . T1 length/width at posterior margin: $0.65 / 0.40 \mathrm{~mm}$. T2 length/width at posterior margin: $0.21 \mathrm{~mm} / 0.80 \mathrm{~mm}$.

Distribution. UNITED STATES, Arizona, Patagonia Mountains, 1,676 m.a.s.l.

Biology. Unknown.

Molecular data. The holotype rendered a partial DNA barcode (164 base pairs), the sequence page in BOLD is HYCNE1802-11.

Etymology. Named after the Madrean sky island ranges in Arizona. The Madrean sky islands are pine-oak woodlands found at higher elevations in Mexico, Arizona and New Mexico (USA); they are surrounded at lower elevations by the Sonoran and Chihuahuan deserts, and are important because of their endemism and relict populations.

\section{Promicrogaster rondeau Fernandez-Triana, sp. nov.} http://zoobank.org/8064D59E-A217-48F9-BFB6-752078A4540F Fig. 7 A-F

Material examined. Holotype. CANADA $\bullet+$, CNC; Ontario; Rondeau Provincial Park; 12 Aug 1982; Sand dunes; M. Sharkey leg; CNC666701. 

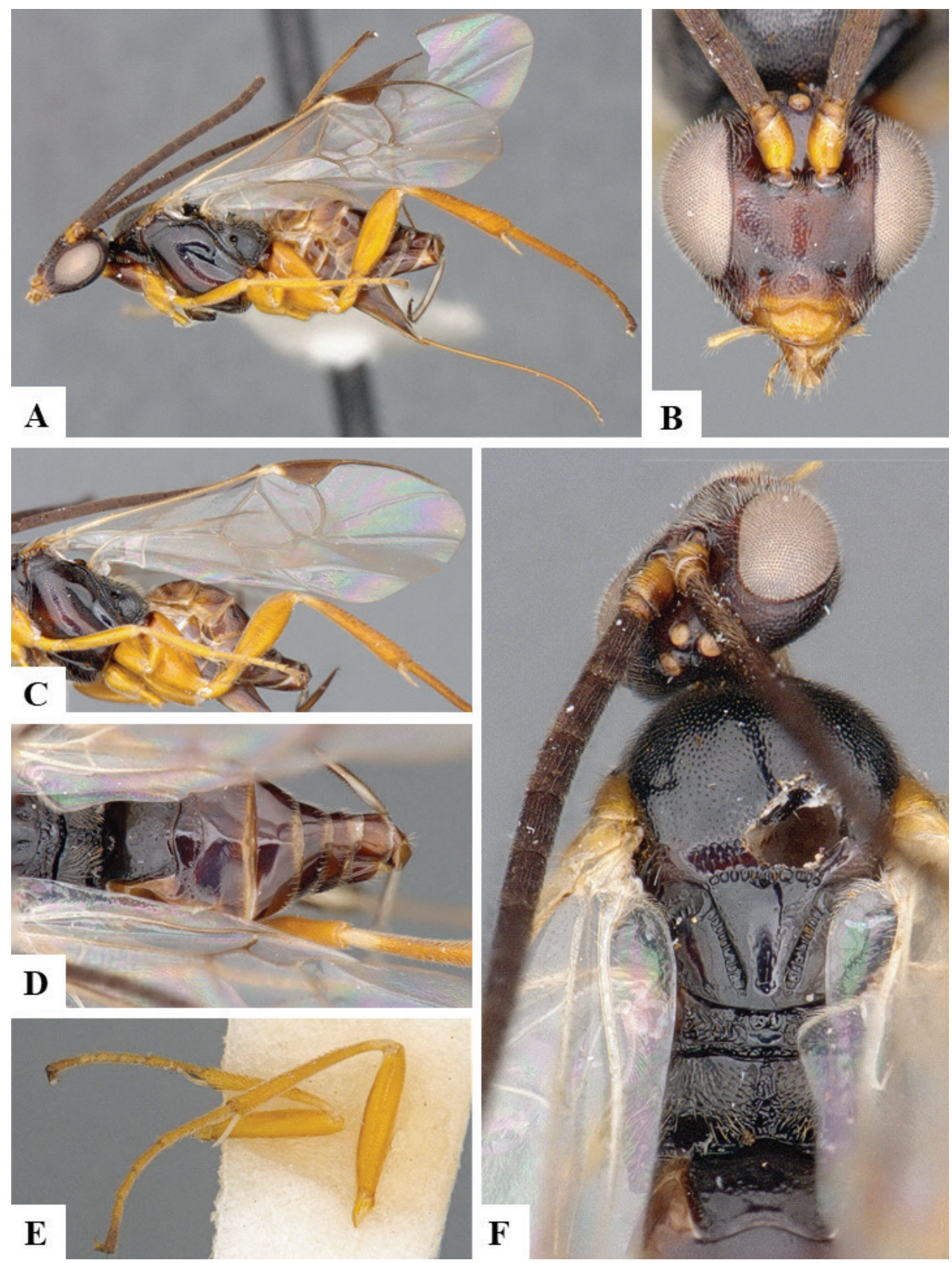

$\mathbf{F}$

Figure 6. Promicrogaster madreanensis, female holotype. A Habitus, lateral $\mathbf{B}$ head, frontal $\mathbf{C}$ fore wing $\mathbf{D}$ propodeum and metasoma, dorsal $\mathbf{E}$ middle legs $\mathbf{F}$ head and mesosoma, dorsal.

Diagnosis. This species is morphologically similar to four species described from Costa Rica (ACG). Apart from the disparate geographical distribution, there are several differences between them, which are best shown in Table 2 below. 
Table 2. Differences between Promicrogaster rondeau (from Canada) and four morphologically related species from Costa Rica. OOL- Ocular-ocellar line; POL- Posterior ocellar line; POD- Posterior ocellus diameter; L- Length. All measurements in mm.

\begin{tabular}{l|c|c|c|c|c|c|c|c|c}
\hline \multicolumn{1}{c|}{ Species } & OOL & POL & POD & $\begin{array}{c}\text { Meta- } \\
\text { coxa L }\end{array}$ & $\begin{array}{c}\text { Meta-femur } \\
\mathbf{L}\end{array}$ & Meta-tibia L & Body L & Fore wing L & $\begin{array}{c}\text { Ovipositor } \\
\mathbf{L}\end{array}$ \\
\hline P. rondeau & $\mathbf{0 . 1 4}$ & $\mathbf{0 . 0 7}$ & 0.05 & $\mathbf{0 . 4 4}$ & $\mathbf{0 . 5 1}$ & $\mathbf{0 . 6 5}$ & 2.20 & 2.30 & $\mathbf{1 . 3 0}$ \\
\hline$P$. fabiancastroi & $0.08-0.10$ & 0.08 & 0.05 & $0.45-0.47$ & $0.52-0.56$ & $0.65-0.69$ & $1.98-2.14$ & $2.23-2.25$ & $1.40-1.57$ \\
\hline P. eddycastroi & 0.11 & 0.08 & 0.06 & 0.56 & 0.68 & 0.89 & 2.29 & 2.65 & 1.77 \\
\hline P. luismendezi & 0.08 & 0.09 & 0.05 & 0.50 & 0.58 & 0.76 & 2.33 & 2.37 & 1.67 \\
\hline P. naomiduarteae & $0.11-0.14$ & $0.07-0.9$ & 0.06 & $0.47-0.53$ & $0.52-0.61$ & $0.66-0.79$ & $2.33-2.39$ & $2.31-2.47$ & $1.41-1.92$ \\
\hline
\end{tabular}

Description. Head: mostly dark reddish-brown, labrum and mandibles yellow. Flagellomeres: dark brown. Mesosoma: mostly dark reddish-brown laterally, dorsally mostly black (but with faint reddish-brown spots on posterolateral margins of anteromesoscutum). Tegula: yellow. Metasoma (dorsally): mostly dark brown to black. Metacoxa: brown. Malar distance: less than $0.2 \times$ eye length. Fore wing areolet: absent. T1 sculpture: mostly sculptured. T2 sculpture: mostly sculptured, except for small, smooth central area. Body length: $2.20 \mathrm{~mm}$. Fore wing length: $2.30 \mathrm{~mm}$. Ovipositor length: $1.30 \mathrm{~mm}$. Ocular-ocellar line: $0.14 \mathrm{~mm}$. Interocellar distance: $0.07 \mathrm{~mm}$. Posterior ocellus diameter: $0.05 \mathrm{~mm}$. Metacoxa length: $0.44 \mathrm{~mm}$. Metafemur length: $0.51 \mathrm{~mm}$. Metatibia length: $0.65 \mathrm{~mm}$. T1 length/width at posterior margin: $0.31 \mathrm{~mm} /$ $0.17 \mathrm{~mm}$. T2 length/width at posterior margin: $0.08 \mathrm{~mm} / 0.32 \mathrm{~mm}$.

Distribution. CANADA, Ontario, Rondeau Provincial Park.

Biology. Unknown.

Molecular data. Not available.

Etymology. Named after the type locality, in recognition of the natural values and uniqueness of the Rondeau Provincial Park.

\section{Promicrogaster virginianus Fernandez-Triana, sp. nov.}

http://zoobank.org/BF705DDC-8701-4A84-AB9F-38B737C9C39A

Fig. 8 A-E

Material examined. Holotype. UNITED STATES • + , USNM; Virginia; 28 Aug 1884; CNC666702.

Diagnosis. Among the darker coloured species (with uniformly dark antenna and entirely dark brown propleuron), with small areolet in the fore wing and tegula and wing base yellow, this species can be distinguished by its relatively small size (body and fore wing length $2.5-2.6 \mathrm{~mm}$ ) and smaller ocelli (ocular-ocellar line $2.6 \times$ as long as posterior ocellus diameter). All other species with dark colouration are either substantially larger (body and fore wing length at least $3.3 \mathrm{~mm}$, usually more), and/or have larger ocelli, lack an areolet or have some other differences (as stated in the key above).

Description. Head: mostly reddish-brown, labrum and mandibles yellow. Flagellomeres: brown. Mesosoma: reddish-brown. Tegula: yellow. Metasoma (dorsally): mostly reddish-brown. Metacoxa: mostly brown. Malar distance: less than $0.2 \times$ eye length. Fore 


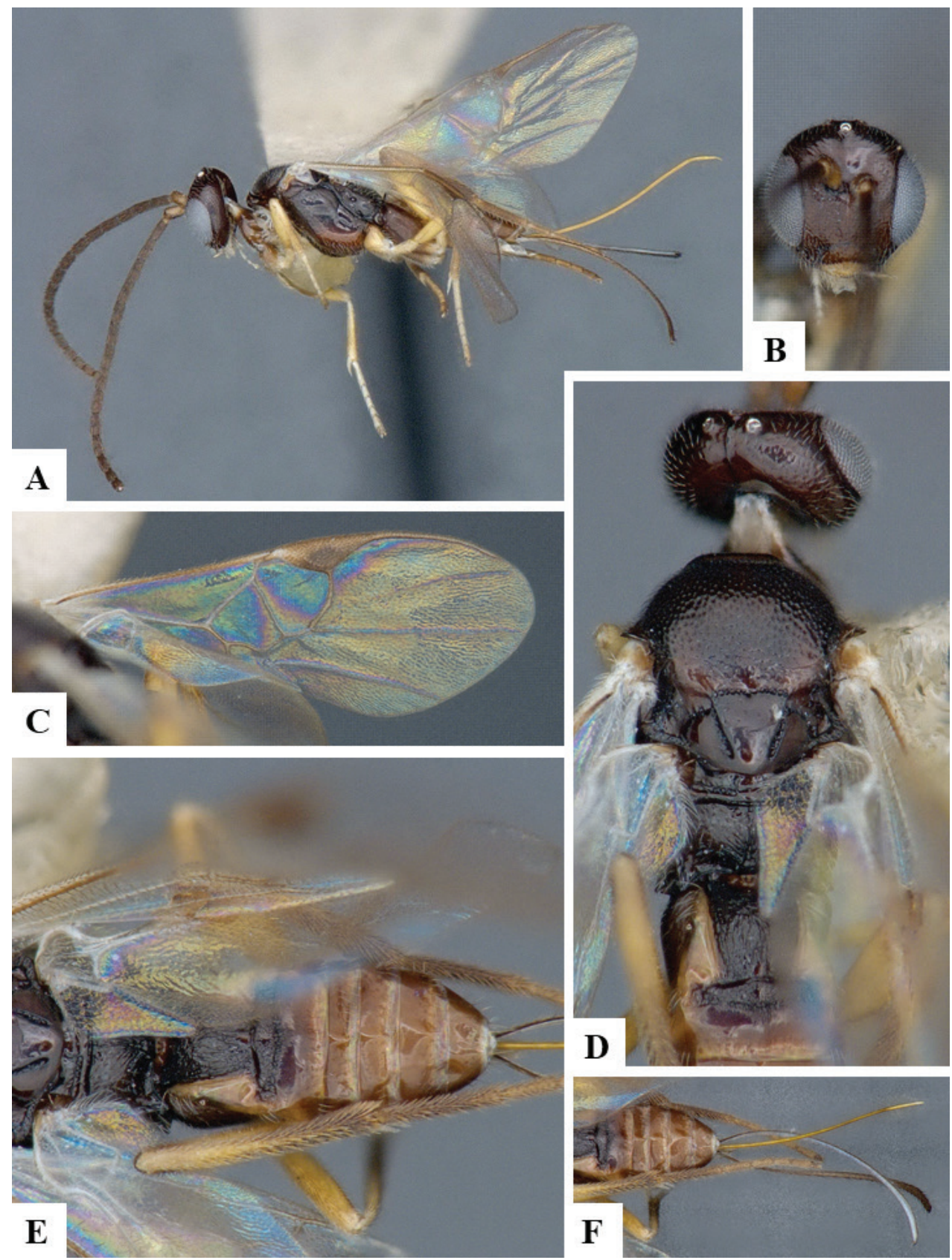

Figure 7. Promicrogaster rondeau, female holotype. A Habitus, lateral B head, frontal $\mathbf{C}$ fore wing $\mathbf{D}$ head and mesosoma, dorsal $\mathbf{E}$ propodeum and metasoma, dorsal $\mathbf{F}$ ovipositor.

wing areolet: present. T1 sculpture: : anterior 0.4 smooth, posterior 0.6 sculptured. T2 sculpture: mostly sculptured. Body length: $2.60 \mathrm{~mm}$. Fore wing length: approximately $2.80 \mathrm{~mm}$. Ovipositor length: $2.30 \mathrm{~mm}$. Ocular-ocellar line: $0.17 \mathrm{~mm}$. Interocellar 

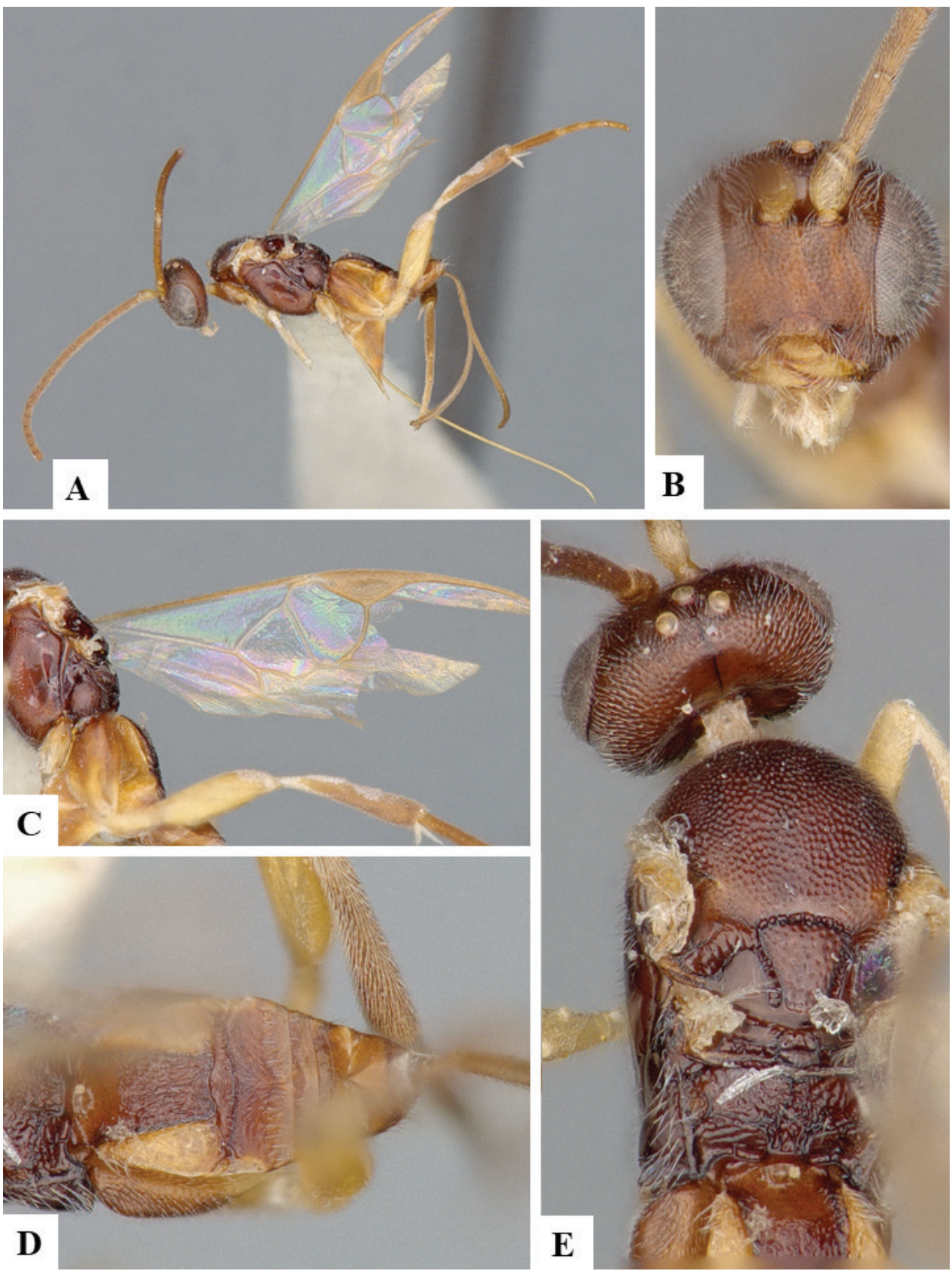

D

Figure 8. Promicrogaster virginianus, female holotype. A Habitus, lateral B head, frontal $\mathbf{C}$ fore wing D propodeum and metasoma, dorsal E head and mesosoma, dorsal.

distance: $0.08 \mathrm{~mm}$. Posterior ocellus diameter: $0.07 \mathrm{~mm}$. Metacoxa length: $0.65 \mathrm{~mm}$. Metafemur length: $0.75 \mathrm{~mm}$. Metatibia length: $0.90 \mathrm{~mm}$. T1 length/width at posterior margin: $0.43 \mathrm{~mm} / 0.18 \mathrm{~mm}$. T2 length/width at posterior margin: $0.08 \mathrm{~mm} / 0.38 \mathrm{~mm}$. 


\section{Distribution. UNITED STATES, Virginia. \\ Biology. Unknown. \\ Molecular data. Not available.}

Etymology. Named after the state where it was collected.

Notes. This is the oldest known specimen of Promicrogaster, having been collected 135 years ago.

\section{Acknowledgements}

I am very grateful for the editorial work of Gavin Broad (Natural History Museum, London, UK) and the review of Mark Shaw (National Museums of Scotland, Edinburgh) which significantly improved the final version of the manuscript. This work was supported by project 3199 "Systematics of beneficial arthropods in support of resilient agroecosystems", Agriculture and Agri-Food Canada.

\section{References}

Fernández-Triana J, Whitfield J, Rodriguez J, Smith M, Janzen D, Hajibabaei M, Burns J, Solis A, Brown J, Cardinal S, Goulet H, Hebert P (2014a) Review of Apanteles sensu stricto (Hymenoptera, Braconidae, Microgastrinae) from Area de Conservación Guanacaste, northwestern Costa Rica, with keys to all described species from Mesoamerica. ZooKeys 383: 1-565. https://doi.org/10.3897/zookeys.383.6418

Fernández-Triana JL, Whitfield JB, Smith MA, Hallwachs W, Janzen DH (2014b) Revision of the neotropical genus Sendaphne Nixon (Hymenoptera, Braconidae, Microgastrinae). Journal of Hymenoptera Research 41: 1-29. https://doi.org/10.3897/JHR.41.8586

Fernández-Triana J, Boudreault C, Dapkey T, Smith MA, Rodriguez J, Hallwachs W, Janzen DH (2016) Revision of the genus Promicrogaster (Hymenoptera, Braconidae, Microgastrinae) from Area de Conservación Guanacaste, Costa Rica, with a key to all species previously described from Mesoamerica. Journal of Hymenoptera Research 50: 25-79. https:// doi.org/10.3897/JHR.50.8220

Hebert PDN, Cywinska A, Ball SL, deWaard JR (2003) Biological identifications through DNA barcodes. Proceedings of the Royal Society B 270:313-321.https://doi.org/10.1098/ rspb.2002.2218

Huber JT, Sharkey MJ (1993) Structure. In: Goulet H, Huber JT (Eds) Hymenoptera of the world: an identification guide to families. Agriculture Canada Research Branch, Monograph No. 1894E, Ottawa, Canada, 13-59.

Karlsson D, Ronquist F (2012) Skeletal morphology of Opius dissitus and Biosteres carbonarius (Hymenoptera: Braconidae), with a discussion of terminology. PLoS ONE 7(4): e32573. https://doi.org/10.1371/journal.pone.0032573

Mason WRM (1981) The polyphyletic nature of Apanteles Foerster (Hymenoptera: Braconidae): A phylogeny and reclassification of Microgastrinae. Memoirs of the Entomological Society of Canada, Ottawa, 147 pp. https://doi.org/10.4039/entm113115fv 
Quicke DLJ (2015) The Braconid and Ichneumonid Parasitoid Wasps: Biology, Systematics, Evolution and Ecology. Willey-Blackwell, 704 pp. https://doi.org/10.1002/9781118907085

Ratnasingham S, Hebert PDN (2007) BOLD: The Barcode of Life Data System [www.barcodinglife.org]. Molecular Ecology Notes 7: 355-364. https://doi.org/10.1111/j.14718286.2007.01678.x

Whitfield JB (1997) Subfamily Microgastrinae. In: Wharton RA, Marsh PM, Sharkey MJ (Eds) Manual of the New World genera of the family Braconidae (Hymenoptera). Special Publication No. 1, International Society of Hymenopterists, Washington, DC, 333-364. 